\title{
La grammaticalisation de Meillet, un siècle plus tard
}

Paul Bessler, Université de Toronto

\section{Introduction}

Presqu'un siècle après sa parution, l'article du célèbre linguiste français Antoine Meillet (1912), article où on trouve le premier emploi du terme grammaticalistion, est l'un des travaux les plus cités par les linguistes qui s'intéressent à l'évolution des langues au niveau morphosyntaxique. Or, les choses n'ont pas toujours été ainsi, et malgré l'importance des idées développées par Meillet dans cet article, il est resté pendant presque tout le vingtième siècle sous l'ombre d'autres développements théoriques en linguistique. Ce n'est que depuis une vingtaine d'années que les linguistes recommencent à s'y intéresser. Le but du présent article est donc de considérer les idées présentées dans ce travail de Meillet à la lumière des principaux développements théoriques du vingtième siècle, afin de mieux comprendre leur valeur à l'époque moderne.

\section{La grammaticalisation de Meillet}

Le travail de Meillet consiste en une présentation de plusieurs exemples de ses recherches sur l'histoire des structures grammaticales du français. Meillet conclut que seuls deux processus peuvent créer une forme grammaticale nouvelle. Le premier est l'innovation analogique, dont un exemple typique serait l'emploi du paradigme de conjugaison dit « du premier groupe » (soit -e, -es, -e, -ons, -ez, -ent) lors de la création d'un néologisme verbal. Le deuxième processus, la grammaticalisation, est défini par Meillet comme « le passage d'un mot autonome au rôle d'élément grammatical » (385). Un exemple classique de ce phénomène, et que Meillet présente en détail à titre illustratif, est le substantif pas, qui, à côté de substantifs comme point, mie(tte) et goutte, était utilisé dès le moyen âge pour renforcer le ne de négation en français. À l'origine, une phrase comme Je ne marche pas n'était donc qu'une variante plus expressive de la phrase Je ne marche. Au cours des siècles, la prononciation de ne est devenue de plus en plus faible et l'emploi de pas est devenu de plus en plus fréquent, de sorte qu'aujourd'hui, c'est pas plutôt que ne qui est considéré comme le mot grammatical qui indique la négation, et ce 
mot grammatical n'a plus qu'une relation homonymique avec le substantif dont il est issu. La deuxième étape de ce processus est l'affixation. Selon Meillet, un mot grammatical, ayant perdu presque toute sa valeur sémantique, a tendance à se prononcer avec de moins en moins de force, de sorte qu'il finit souvent par s'attacher phonétiquement au mot précédent ou suivant. À notre connaissance, il n'existe aucune variété du français où l'adverbe de négation pas se réalise comme un affixe, mais si on considère la structure équivalente en anglais, on remarque qu'il y a plusieurs constructions où l'adverbe de négation not a été réduit au statut de suffixe, comme par exemple can not > cannot > can't, do not > don't, etc.

Comme nous l'avons déjà souligné, ce travail de Meillet n'a pas été bien reçu par les linguistes du vingtième siècle. Une raison en est que les deux conclusions les plus importantes que tire Meillet de son analyse, sont également les idées les plus contestées. La première de ces conclusions est que la grammaticalisation est un processus strictement unidirectionnel. Autrement dit, le processus mot lexical > mot grammatical > affixe grammatical n'a jamais lieu dans le sens opposé. La deuxième conclusion est que la grammaticalisation résulte du besoin constant qu'éprouvent les locuteurs de renforcer ou de rendre plus expressifs leurs énoncés, et qu'il s'agit donc d'un processus qui caractérise le langage humain en général. Autrement dit, la grammaticalisation est un processus qui est constamment en train de se produire dans toutes les langues du monde. À propos de l'adverbe de négation pas, par exemple, Meillet remarque que puisqu'il s'agit aujourd'hui d'un mot grammatical qui a perdu sa valeur expressive, les francophones sont obligés de recourir à de nouvelles tournures pour rendre leurs phrases négatives plus expressives : «On sait comment pas a perdu, dans les phrases où il était un accessoire de la négation, tout son sens propre [...], comment pas est devenu à lui seul un mot négatif, [...] et comment par suite le pas français n'est plus expressif à son tour et appelle un nouveau renforcement [...] ; on est amené à dire pas du tout, absolument pas, ou à recourir à des tours tout nouveaux » $(393)^{1}$. Ces deux caractéristiques de la conception de la grammaticalisation de Meillet, c'est-à-dire l'unidirectionnalité et son caractère universel, étaient à l'époque considérées comme problématiques dans la mesure où elles semblaient accorder au modèle un pouvoir de prédiction. En effet, il était généralement 
considéré comme non seulement inutile, mais aussi méthodologiquement dangereux, d'essayer de prédire les changements qui auraient lieu dans une langue donnée.

Un deuxième facteur qui a contribué à «l'assombrissement» du travail de Meillet, est la publication, moins de cinq ans plus tard, du célèbre Cours de linguistique générale de Ferdinand de Saussure (1916). Saussure y établit une distinction nette entre linguistique synchronique et diachronique et remarque que la linguistique ne dépassera le stade de simple outil taxonomique pour devenir une véritable discipline de recherche scientifique que si les linguistes se concentrent sur le fonctionnement synchronique des langues. Cette idée a été renforcée encore davantage lors de la publication, de l'autre côté de l'Atlantique cette fois-ci, de Syntactic Structures de Noam Chomsky (1957), marquant ainsi les débuts de l'école générativiste. Selon Chomsky, l'objet primaire de recherche du linguiste doit être non pas la langue elle-même, mais bien plutôt les connaissances qu'en possède le locuteur. Selon sa «Théorie des principes et paramètres », ces connaissances n'ont que deux sources possibles : certaines sont innées, d'autres viennent des données auxquelles l'enfant est exposé durant le processus d'acquisition. Comme l'enfant qui acquiert sa langue maternelle n'a pas accès aux données historiques, elles ne sont d'aucune valeur dans la description des connaissances linguistiques.

Nous sommes d'avis qu'il y a lieu de considérer ces deux critiques du travail de Meillet plus en détail, afin de déterminer jusqu'à quel point elles sont justifiées. Pour ce qui est du problème de l'unidirectionnalité, il faut d'abord noter que Meillet n'exclut pas la possibilité de l'existence d'un processus du type affixe grammatical > mot grammatical > mot lexical ; il affirme simplement qu'un tel processus n'a jamais été attesté. Deuxièmement, si Meillet conclut que le processus mot lexical > mot grammatical > affixe grammatical représente une tendance générale qui caractérise l'évolution des langues, il ne prétend aucunement pouvoir prédire quels changements auront lieu dans une langue donnée à un moment donné de son évolution. Il est aussi intéressant de noter à cet égard que toutes les théoriques linguistiques font une distinction plus ou moins nette entre éléments lexicaux comme les substantifs, les verbes, les adjectifs, etc., et éléments grammaticaux comme les pronoms, les articles, etc. L'une des différences fondamentales entre ces deux classes d'éléments est que les unités lexicales appartiennent à des listes ouvertes auxquelles les langues ajoutent régulièrement des 
néologismes, tandis que les unités grammaticales appartiennent à des listes fermées auxquelles les langues ne peuvent pas ajouter de nouvelles unités. Cette caractéristique des unités grammaticales soulève naturellement la question de leur origine. Meillet présente à cet égard une analyse de l'immense travail de Franz Bopp sur l'histoire des langues indo-européennes (cf. Bopp 1856), et remarque que «Après une cinquantaine d'années d'essais infructueux en ce genre, on a compris que l'origine première des formes grammaticales est hors de nos prises. » (Meillet 385) Meillet conclut ainsi que la seule origine possible des unités grammaticales est la transformation d'unités lexicales :

Sans doute, quand un mot passe au rôle de forme grammaticale, on peut dire, en un certain sens, qu'il a été créé une forme, mais cette création a lieu à l'intérieur d'une langue qui offre déjà une organisation grammaticale complète, et cette création ne donne pas une idée de ce qui a pu se passer en un temps où il n'existait aucun commencement d'organisation grammaticale. Toutefois on peut retenir que, l'analogie étant par définition exclue de la première origine des formes, le seul procédé qui reste est l'attribution progressive d'un rôle grammatical à des mots autonomes ou à des manières de grouper les mots. (386)

Il nous paraît donc tout à fait plausible de proposer que le seul changement «abrupte » qui puisse se produire dans une langue est la création d'un nouveau mot lexical. Les changements de prononciation et de sens que subissent les mots se produisent toujours lentement, sans que les locuteurs de la langue en question en soient conscients. Si cette conception est correcte, on peut conclure que la seule façon de créer un nouveau mot ou affixe grammatical est la modification d'un élément qui existe déjà dans la langue en question. Si on adopte une telle conception de l'évolution des langues, l'unidirectionnalité de la grammaticalisation est non seulement plausible, mais empiriquement bien fondée, et l'argument selon lequel Meillet essaie, à tort, de prédire les changements linguistiques, perd toute sa force.

Pour ce qui est du problème de l'acquisition, il est vrai qu'un enfant qui acquiert sa langue maternelle, n'a pas accès aux données historiques, mais il est tout aussi vrai que les modèles strictement synchroniques du langage, et plus particulièrement le modèle chomskyen, font abstraction d'une caractéristique fondamentale du langage humain. Nous savons définitivement que toutes les langues du monde évoluent constamment et 
que les enfants réussissent, génération après génération, à acquérir leur langue maternelle. La question qui se pose, est donc la suivante : Comment un modèle linguistique peut-il être compatible et avec le concept d'évolution des langues et avec l'acquisition ? Le fait que les modèles strictement synchroniques font abstraction de cette question, ne la rend pas moins importante.

\section{Développements théoriques récents}

Des éléments de réponse à la question de la relation entre l'évolution des langues et l'acquisition que nous venons de soulever, se trouvent peut-être dans des modèles théoriques plus récents, grâce auxquels les linguistes recommencent depuis récemment à prendre au sérieux le modèle de grammaticalisation de Meillet. Un premier développement dans ce sens est le concept de «synchronie dynamique », proposé par Roman Jakobson (1963), étudiant de Meillet. L’idée est qu'on peut considérer une langue à un moment précis de son évolution, tout en étant conscient du fait qu'il y a des structures qui sont en train d'être modifiées : «Pendant un certain temps, le point de départ et le point d'aboutissement de la mutation se trouvent coexister sous la forme de deux couches stylistiques différentes [...] Un changement est donc, à ses débuts, un fait synchronique » (Jakobson 37).

Un deuxième concept qui a été proposé pour résoudre des problèmes comme celui que nous venons de soulever, est la réanalyse. Il existe plusieurs versions de cette théorie dans la documentation ; nous adopterons ici la définition de Ronald Langacker (1977) « [...] change in the structure of an expression or class of expressions that does not involve any immediate or intrinsic modifications of its surface manifestation » (58). Comme le montrent les structures arborescentes ci-dessous, la représentation structurale que les locuteurs d'une langue assignent à une suite de sons, syllabes, mots, etc., peut être modifiée sans qu'il y ait modification de la suite d'unités elle-même. 
1)

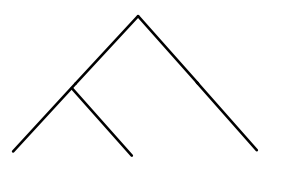

A $\quad$ B $\quad$ C
2)

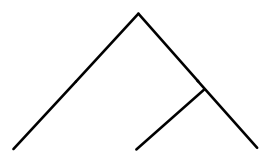

A $\quad$ B $\quad$ C

Un exemple de ce phénomène en français est le syntagme nominal "l'évier", où la séquence article+nom a été réanalysée dans certaines variétés non-standard du français comme un seul nom, soit lévier, donnant lieu au syntagme "le lévier" (cf. Wittmann et Fournier 1982).

Il existe dans la documentation quelques tentatives récentes d'intégrer la grammaticalisation et la réanalyse dans le modèle des principes et paramètres. Selon Ian Roberts et Anna Roussou (2003), " grammaticalization involves the creation of new functional material, either through the reanalysis of existing functional material or through the reanalysis of lexical material »(2). Roberts et Rousseau proposent une version modifiée du modèle des principes et paramètres, et proposent que les changements linguistiques ont lieu comme suit : «A change is initiated when (a population of) learners converge on a grammatical system which differs in at least one parameter value from the system internalized by the speakers whose linguistic behaviour provides the input to the learners. As the younger generation replaces the older one, the change is carried through the speech community » (11). Bien que ce modèle constitue un véritable pas en avant au niveau de l'intégration de faits diachroniques dans la théorie chomskyenne, il paraît que Roberts et Rousseau sont toujours restreints par le formalisme de leur cadre théorique. Plus spécifiquement, l'idée que les changements linguistiques ne peuvent avoir lieu qu'au niveau paramétrique, ainsi que l'idée que les changements doivent se produire au cours d'une seule génération, donnent lieu à un modèle qui est bien trop rigide pour rendre compte d'un changement comme celui de l'adverbe de négation pas que nous avons présenté ci-haut. Il nous paraît qu'au moins deux modifications sont nécessaires afin de permettre à ce modèle de rendre compte de tels changements morphosyntaxiques. Premièrement, il faut permettre que les changements 
linguistiques aient lieu non seulement au niveau paramétrique, mais aussi au niveau lexical. Deuxièmement, il faut modifier la définition de la réanalyse pour inclure des changements qui ont lieu au cours de plus d'une génération. Pour faire ceci, nous proposons une modification de la définition de la réanalyse, selon laquelle il y a une étape d'« ambiguïté » entre l'ancienne structure et la nouvelle.

1)

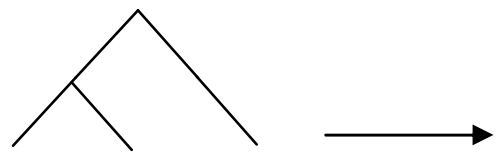

A $\quad$ B $\quad$ C
2)

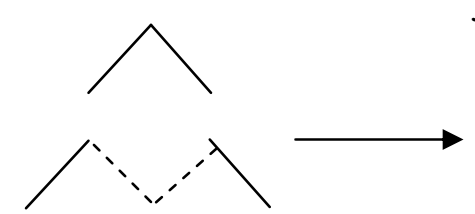

A $\quad$ B $\quad$ C
3)

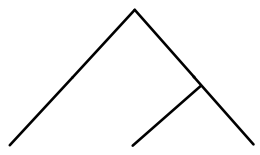

A $\quad$ B $\quad$ C

Si nous reprenons l'exemple de l'adverbe de négation pas, nous pouvons dire que, si l'arbre (1) représente la structure où pas n'était qu'un substantif qui renforçait le ne de négation et que l'arbre (3) représente la structure actuelle, où pas est un adverbe de négation qui n'a plus de lien avec le substantif, l'arbre (2) correspond à la période historique où le ne de négation se prononçait avec de moins en moins d'intensité, et où le substantif pas s'employait de plus en plus fréquemment, de sorte que pas commençait à acquérir certaines propriétés adverbiales. Ces changements de prononciation et de sens ont permis à la longue aux locuteurs d'assigner la structure (3) aux phrases négatives. Il faudrait évidemment une analyse beaucoup plus détaillée de l'évolution de cette structure afin de donner une description complète du passage de la structure (1) à la structure (2) et de la structure (2) à la structure (3), mais même l'analyse superficielle que nous avons présentée ici suggère qu'il est possible d'intégrer l'évolution des langues et l'acquisition. Plus spécifiquement, il nous parait tout à fait plausible de postuler qu'une génération d'enfants qui apprennent une construction ayant la structure (1), la réanalysent comme ayant la structure (2), et qu'une génération subséquente apprennent cette construction ayant la structure (2) et la réanalysent comme ayant la structure (3). 


\section{Conclusion}

Nous ne prétendons nullement avoir résolu dans ce travail l'épineux problème de la relation entre l'évolution des langues et l'acquisition. Nous espérons plutôt avoir montré que la contribution de Meillet à cet aspect de la linguistique, est tout aussi valable qu'elle l'était il y a un siècle. Nous espérons également avoir créé, en ajoutant au modèle de Meillet quelques outils théoriques plus récents, un modèle qui servira de point de départ pour des recherches ultérieures dans ce domaine.

\section{Bibliographie}

Bopp, Franz. Vergleichende Grammatik des Sanskrit, Send, Griechischen, Lateinischen, Litauischen, Altslavischen, Gothischen und Deutschen, Berlin : Dümmler, 1856.

Jakobson, Roman. Essais de linguistique générale. Paris : Minuit, 1963.

Langacker, Ronald. «Syntactic Reanalysis ». Mechanisms of Syntactic Change. Dir. Charles Li. Austin : U of Texas P, 1977. 57-139.

Meillet, Antoine. «L'évolution des formes grammaticales ». Scientia 12 (1912) : 384400.

Roberts, Ian et Anna Roussou. Syntactic Change - A Minimalist Approach to Grammaticalization. Cambridge : Cambridge UP, 2003.

Saussure, Ferdinand de. Cours de linguistique générale. Paris : Payot, 1916.

Wittmann, Henri et Robert Fournier. «L'agglutination nominale en français colonial (populaire et créole) ». Revue québécoise de linguistique théorique et appliquée 2 (1982) : 185-209.

\footnotetext{
Notes

${ }^{1}$ Il y a lieu de se demander si Meillet était conscient de l'existence de l'adverbe pantoute du français canadien populaire, qui constitue un exemple parfait du processus dont Meillet discute.
} 